\title{
Novel Strategy to Improve End-Organ Function With Pulsatile Cardiopulmonary Bypass
}

\author{
Pavan Atluri, MD; John W. MacArthur, MD
}

$\mathbf{T}$ he advent of cardiopulmonary bypass (CPB) has enabled cardiovascular surgeons to perform complex, life-saving procedures with relative ease while protecting end-organ function. Altering the normal pulsatile blood flow to non-pulsatile continuous flow has raised concerns over potential detrimental hemodynamic and physiologic consequences. Concerns over long-term non-pulsatile flow drove the design for the original pulsatile mechanical circulatory support devices. Unfortunately, the increased number of mobile parts required to generate pulsatile flow has rendered this technology inadequate for destination mechanical assistance. ${ }^{1}$ Second- and third-generation continuous flow left ventricular assist devices (LVAD) such as the HeartMate II (Thoratec, Pleasanton, CA, USA) and HVAD (HeartWare, Framingham, MA, USA) use minimal wearable parts, which has decreased concerns over long-term durability and pump failure. ${ }^{2}$ Over the past several years we have witnessed thousands of patients survive for years with preserved end-organ function in the presence of non-pulsatile LVAD flow. Although patients are living longer with these non-pulsatile flow devices, it has become evident that there is a new subset of pathology that is germane to continuous flow. The advent of de novo aortic insufficiency, gastrointestinal and cerebrovascular arteriovenous malformations, and acquired von Willebrand disease has been witnessed in this patient population. ${ }^{3}$ Though these long-term issues are not directly applicable to $\mathrm{CPB}$, because of its acuity, they stress the importance of pulsatile flow in normal circulatory function.

\section{Article p1121}

It has been known for years that CPB initiates an intense inflammatory and coagulopathic state. The ability to ameliorate this response could be quite beneficial to postoperative patients' outcomes and the preservation of organ function. Numerous studies have clearly documented the release of inflammatory cytokines, growth factors and endothelial cell activation with the institution of CPB. This deleterious sequence of events appears to be related to multiple factors, including ischemiareperfusion injury associated with cardioplegic arrest, interaction between blood and the artificial circuitry of the CPB circuit, and endothelial cell activation with subsequent release of cytokines. Endothelial activation is associated with eNOS activation, upregulation of Akt and ERK 1/2 pathways, and upregulation of endothelial cytokines and inflammatory markers (ie, VEGF, MCP-1, vWF, ACE). ${ }^{4-6}$ Moreover, the systemic manifestation of this post-CPB systemic inflammatory response (SIRS) can be quite pronounced. ${ }^{7}$ From a pulmonary standpoint, this manifests as increased pulmonary resistance and poor alveolar oxygenation, necessitating prolonged ventilator support. ${ }^{8}$ The SIRS response can be profound enough to mediate hepatic and renal failure.

Recent studies have demonstrated that reducing the length of tubing that is used in the CPB circuit (ie, "miniaturized CPB") can result in reduction in the inflammatory response and SIRS that ensue. ${ }^{9}$ The thought that revascularizing the heart without the use of a CPB circuit minimizes the inflammatory cascade has prompted surgeons to pursue off-pump coronary artery bypass grafting (OPCAB). Numerous clinical trials have attempted to demonstrate a clinical benefit with OPCAB as compared with standard on-bypass $\mathrm{CABG}$, but have failed to show a clear cut benefit of 1 technique over the other. Though a clinical benefit has not been demonstrated, a significant reduction in the inflammatory cascade that has been associated with using the CPB circuit has been clearly demonstrated with beating heart coronary revascularization vs. standard arrested heart on-pump coronary artery bypass. ${ }^{10-12}$ But, given concerns over the quality of revascularization, low ejection fraction, and the learning curve associated with good outcomes, many believe that on-pump CABG may still be a better strategy for revascularization. Additionally, CPB remains a must for cardiovascular surgical repair of structural heart disease (ie, valvular, aortic). As such, we must continue to search for means of minimizing inflammation and stress associated with CPB.

In this issue of the Journal, Drs Serraino and colleagues have presented the results of their very interesting study of the use of CPB with continuous intra-aortic balloon pump counter pulsation to generate a pulsatile CPB circuit. ${ }^{13}$ The authors conducted a randomized, prospective clinical trial in which 501 CABG patients were randomized to either standard non-pulsatile CPB or pulsatile CPB. They investigated both activation of the inflammatory pathway and injury to end-organs (liver, kidney, lung). They demonstrate very clear improvements in endothelial activation and end-organ function. There were significant improvements in liver function (coagulation, metabolic parameters), lower rates of renal failure, and improved alveolar gas exchange. The investigators should be commended for their novel study. Their data raise the question of whether we should change our current paradigm and investigate

The opinions expressed in this article are not necessarily those of the editors or of the Japanese Circulation Society.

Received March 8, 2012; accepted March 8, 2012; released online March 27, 2012

Division of Cardiovascular Surgery, Department of Surgery, Perelman School of Medicine, University of Pennsylvania, Philadelphia, PA, USA

Mailing address: Pavan Atluri, MD, Assistant Professor of Surgery, Division of Cardiovascular Surgery, Department of Surgery, University of Pennsylvania, Philadelphia, PA 19104, USA. E-mail: pavan.atluri@uphs.upenn.edu

ISSN-1346-9843 doi:10.1253/circj.CJ-12-0294

All rights are reserved to the Japanese Circulation Society. For permissions, please e-mail: cj@j-circ.or.jp 
means of generating pulsatile flow during CPB. There is no question that we have had excellent clinical results with the current CPB circuits, but the results presented by Drs Serraino and colleagues bring into question whether we should be seeking to further improve our techniques.

This strategy, though proven beneficial with coronary revascularization, may have a more beneficial effect in cases of traditionally longer CPB times: aortic surgery, and complex valve and coronary surgery. Moreover, these findings raise the question of whether patients supported with extracorporeal membrane oxygenation would benefit from concomitant insertion of an intra-aortic balloon pump with continuous aortic counter pulsation in order to provide a pulsatile flow. Clearly though, despite the very encouraging results of this study, further investigation should be performed in order to determine the clinical benefit. Additional multi-institutional clinical trials should be performed.

\section{References}

1. Slaughter MS, Rogers JG, Milano CA, Russell SD, Conte JV, Feldman $\mathrm{D}$, et al. Advanced heart failure treated with continuous-flow left ventricular assist device. N Engl J Med 2009; 361: 2241-2251.

2. Fitzpatrick III JR, Woo YJ. Mechanical circulatory assistance. Circ J 2011; 75: 38-46.

3. Hatano M, Kinugawa K, Shiga T, Kato N, Endo M, Hisagi M, et al. Less frequent opening of the aortic valve and a continuous flow pump are risk factors for postoperative onset of aortic insufficiency in patients with a left ventricular assist device. Circ J 2011; 75: 1147 1155 .

4. Onorati F, Rubino AS, Cuda A, Foti D, Sica V, Santini F, et al. Impact of endothelial activation on infective and inflammatory complications after cardiac surgery in type II diabetes mellitus. Int J Artif Organs 2011; 34: 469-480.
5. Fischer UM, Klass O, Cox CS, Stock U, Antonyan A, Brixius K, et al. Regulation of endothelial nitric oxide synthase (eNOS) in myocardium subjected to cardioplegic arrest. Thorac Cardiovasc Surg 2009; 57: 379-385.

6. Panagiotopoulos I, Palatianos G, Michalopoulos A, Chatzigeorgiou A, Prapas S, Kamper EF. Alterations in biomarkers of endothelial function following on-pump coronary artery revascularization. J Clin Lab Anal 2010; 24: 389-398.

7. Sobieski MA 2nd, Graham JD, Pappas PS, Tatooles AJ, Slaughter MS. Reducing the effects of the systemic inflammatory response to cardiopulmonary bypass: Can single dose steroids blunt systemic inflammatory response syndrome? ASAIO J 2008; 54: 203-206.

8. Kagawa H, Morita K, Nagahori R, Shinohara G, Kinouchi K, Hashimoto K. Prevention of ischemia/reperfusion-induced pulmonary dysfunction after cardiopulmonary bypass with terminal leukocyte-depleted lung reperfusion. J Thorac Cardiovasc Surg 2010; 139: 174-180.

9. Bical OM, Fromes Y, Gaillard D, Fischer M, Ponzio O, Deleuze P, et al. Comparison of the inflammatory response between miniaturized and standard CPB circuits in aortic valve surgery. Eur J Cardiothorac Surg 2006; 29: 699-702.

10. Bayram H, Erer D, Iriz E, Zor MH, Gulbahar O, Ozdogan ME. Comparison of the effects of pulsatile cardiopulmonary bypass, non-pulsatile cardiopulmonary bypass and off-pump coronary artery bypass grafting on the inflammatory response and S-100beta protein. Perfusion 2012; 27: 56-64.

11. Stassano P, Di Tommaso L, Monaco M, Iesu S, Brando G, Buonpane $\mathrm{S}$, et al. Myocardial revascularization by left ventricular assisted beating heart is associated with reduced systemic inflammatory response. Ann Thorac Surg 2009; 87: 46-52.

12. Onorati F, Rubino AS, Nucera S, Foti D, Sica V, Santini F, et al. Offpump coronary artery bypass surgery versus standard linear or pulsatile cardiopulmonary bypass: Endothelial activation and inflammatory response. Eur J Cardiothorac Surg 2010; 37: 897-904.

13. Serraino GF, Marsico R, Musolino G, Ventura V, Gulletta E, Santè P, et al. Pulsatile cardiopulmonary bypass with intra-aortic balloon pump improves organ function and reduces endothelial activation. Circ $J$ 2012; 76: $1121-1129$. 